\title{
Right Hepatic Lobe Agenesis Combined with Budd-Chiari Syndrome - A Case Report and Literature Review
}

\author{
Ruifang Xu, MD ${ }^{a}$, Ji-Bin Liu, MD ${ }^{b}$, Linxue Qian, MD, PhD ${ }^{a, *}$ \\ ${ }^{a}$ Department of Ultrasound, Beijing Friendship Hospital, Capital Medical University, Beijing, China, ${ }^{b}$ Department of Radiology, Thomas \\ Jefferson University Hospital, Philadelphia, PA, USA.
}

Received April 26, 2020; revision received May 05, 2020; accepted June 08, 2020

\begin{abstract}
Agenesis of the right lobe of the liver is an extremely rare congenital anomaly and usually detected by incidental cross-sectional imaging examination. Budd-Chiari syndrome is a rare cause of portal vein hypertension and hepatic failure, with the characteristic of the hepatic venous outflow tract obstruction. This article presented a clinical case of a 43 years old female diagnosed with agenesis of the right hepatic lobe combined with Budd-Chiari syndrome by ultrasonography (US) and computed tomography $(\mathrm{CT})$ and conducted a literature review for imaging diagnosis of right hepatic lobe agenesis.
\end{abstract}

Key words: Right hepatic lobe agenesis; Budd-Chiari syndrome; Congenital anomaly; Imaging diagnosis

Advanced Ultrasound in Diagnosis and Therapy 2020;03:234-238

DOI: 10.37015/AUDT.2020.200042

\section{Introduction}

Congenital agenesis of a main liver lobe is a rare malformation, in which the left lobe of the liver is affected more often than the right one [1]. The patients are usually asymptomatic, with normal liver function and testing parameters. Before the introduction of cross-sectional imaging technologies, including ultrasonography (US), computed tomography (CT) and magnetic resonance imaging (MRI), congenital agenesis of a main liver lobe is often detected incidentally during laparotomy, autopsy and/ or minimally invasive examinations such as angiography or percutaneous cholangiography [2]. Agenesis of the right lobe of the liver is conceptually defined as the absence of liver tissue on the right side of the gallbladder fossa, with or without preservation of the middle hepatic vein, and without previous disease or surgery [3]. Generally, it is associated with other anatomical alterations, such as hypertension of other liver segments, colonic interposition between liver and diaphragm, right diaphragmatic hernia, portal hypertension, or an anomalously positioned gallbladder [4-5].
Budd-Chiari syndrome (BCS) is a group of disorders featured by hepatic venous outflow tract obstruction (HVOTO) at various levels from the small or large hepatic veins to the supra-hepatic portion of the inferior vena cave (IVC), causing combination of congestive portal hypertension and/or inferior vena cava hypertension, except for sinusoidal obstruction syndrome/veinocclusive disease [6]. BCS can be divided into two groups: asymptomatic forms and symptomatic forms according to the clinical manifestations. About $15 \%$ $20 \%$ of BCS patients are asymptomatic with obvious clinical presentation, in which the major compensatory mechanism is the spontaneous development of large intraor extra-hepatic portal-systemic shunts [7]. However, most patients with BCS are symptomatic, including abdominal pain, ascites, jaundice, hepatomegaly, edema, hepatic encephalopathy and/or gastrointestinal bleeding. We report a case with agenesis of the right hepatic lobe associated with BCS diagnosed by ultrasonography (US) and computed tomography (CT) and conducted a literature review for imaging diagnosis of right hepatic

* Corresponding author: Department of Ultrasound, Beijing Friendship Hospital, Capital Medical University, No. 95, Yongan Road, Xicheng District, Beijing 100050, China.

e-mail:qianlinxue@outlook.com 
lobe agenesis.

\section{Case Report}

A 43-year-old female developed swelling in the lower extremities without obvious inducement ten months ago. About one week later, the lower extremities swelling relieved spontaneously without special treatment. Two months ago, the patient complained of abdominal distension, without abdominal pain, diarrhea, vomiting, melena or jaundice. The patient and her family had no history of liver diseases, had no habit of alcohol drinking, nor blood transfusion. Abdominal computed tomography (CT) of the patient from the local hospital indicated liver cirrhosis and ascites. The patient was then admitted to our hospital for further diagnosis and medical treatment. Physical examination of the patient revealed abdominal enlargement, hepatomegaly, and the liver $5 \mathrm{~cm}$ below xiphoid process by palpation. Laboratory examinations exhibited hemoglobin (HGB) $83 \mathrm{~g} / \mathrm{L}$, WBC $8.01 \times 10^{9} / \mathrm{L}$, platelet (PLT) $284 \times 10^{9} / \mathrm{L}$, ALT $12 \mathrm{U} / \mathrm{L}$, AST $25.1 \mathrm{U} / \mathrm{L}$, prothrombin time (PT) $14.7 \mathrm{~s}$, prothrombin time activity (PTA) $65.8 \%$, and activated partial thromboplastin time (APTT) $29.3 \mathrm{~s}$. The patient had no hepatitis or previous abdominal surgery. However, the patient had complained of abdominal injury history when she was eight years old, that was relieved spontaneously without special treatment.
Abdominal ultrasonography using an ultrasound scanner with a 1-5 MHz convex transducer (iU Elite, Philips, Bothell, WA, USA) showed the absence of liver tissue on the right side of gallbladder with compensatory hypertrophy of left hepatic lobe, rough liver surface, parenchymal echogenicity increased, and no dilation of intrahepatic and extrahepatic bile ducts (Fig. 1A-C). The inner diameter of hepatic vein of the segment $4\left(\mathrm{~S}_{4} \mathrm{~V}\right)$ was $1.0 \mathrm{~cm}$ while no blood flow could be detected at the point close to the inferior vena cava, however, the collateral vessels draining into the left hepatic vein were seen (Fig. 1B). The inner diameter of the middle part of the left hepatic vein (LHV) was about $1.2 \mathrm{~cm}$ and the lumen was thinner closer to the inferior vena cava. The inner diameter of the left hepatic vein at the junction to the inferior vena cava was $0.3 \mathrm{~cm}$ and the peak flow velocity was $183 \mathrm{~cm} / \mathrm{s}$ as monophasic waveform without normal periodic venous plasticity which suggested stenosis (Fig. 1C, D). The lumen of the hepatic veins did not change during the respiration. The right and middle hepatic veins cannot be detected. The size and shape of gallbladder were as usual appearance, with gallbladder wall thickness of $0.5 \mathrm{~cm}$. The gallbladder was located at the right side of the left hepatic lobe and floated in the right subphrenic ascites (Fig. 1A). A large amount of ascites could be seen with the maximum depth of $7.4 \mathrm{~cm}$.
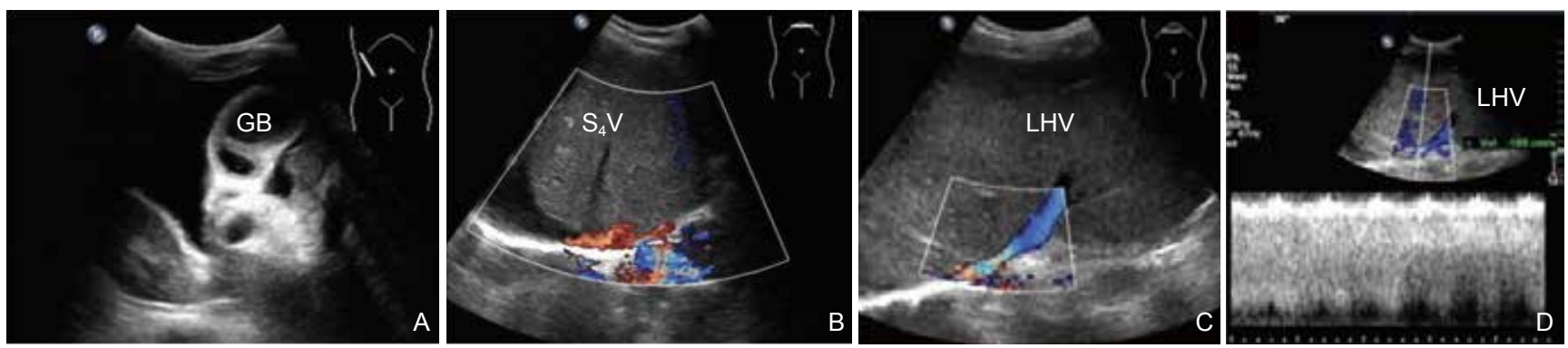

Figure 1 (A) Gallbladder with normal size and shape located on the right side of the left hepatic lobe and floated in the right subphrenic ascites; (B) No blood flow signal could be detected in the S4V at the secondary porta; (C\&D) The lumen was narrow closer to the inferior vena cava of the LHV, and the peak flow velocity was $183 \mathrm{~cm} / \mathrm{s}$ as monophasic waveform without normal periodic venous plasticity. GB, gallbladder; S4V, hepatic vein of the segment 4 ; LHV, left hepatic vein.

Contrast-enhanced computed tomography (CECT) (iCT 256, Philips Medical Systems, Best, The Netherlands) illustrated no liver parenchyma on the right side of the gallbladder fossa, compensatory hypertrophy of left hepatic lobe and complete absence of the right and middle hepatic vein seen. The gallbladder was in a retrohepatic position, floating in the right subphrenic ascites. The liver parenchyma was heterogeneous in appearance, and geographically distributed hepatic congestion was observed during the portal venous phase (Fig. 2A). Only the left hepatic vein and the $\mathrm{S}_{4} \mathrm{~V}$ could be detected. The middle hepatic vein and right hepatic vein were absent. Vascular reconstruction of CT imaging demonstrated complete absence of the right hepatic artery and gallbladder artery originating from the left branch of hepatic artery. No replacement of the right hepatic artery from the superior mesenteric artery could be detected (Fig. 2B). Complete absence of right portal vein branch from the main portal trunk and right hepatic artery from the main hepatic artery were noted (Fig. 2C, D). The inferior vena cava of this case didn't pass through the liver parenchyma on any of the CT 
images but simply contacted with the posterior visceral surface of the left lobe (Fig. 3A, B, C). This patient did further digital subtraction angiography (DSA) for further diagnosis. There was no obvious stenosis of the inferior vena cava seen (Fig. 3D). No obvious enhancement of the hepatic vein or its collateral branches were observed.
The entrance points of the hepatic veins to the inferior vena cava could not be detected. Based on the ultrasound and CT finding and clinical presentation, the diagnosis of this case was made as agenesis of the right hepatic lobe combined with Budd-Chiari syndrome.
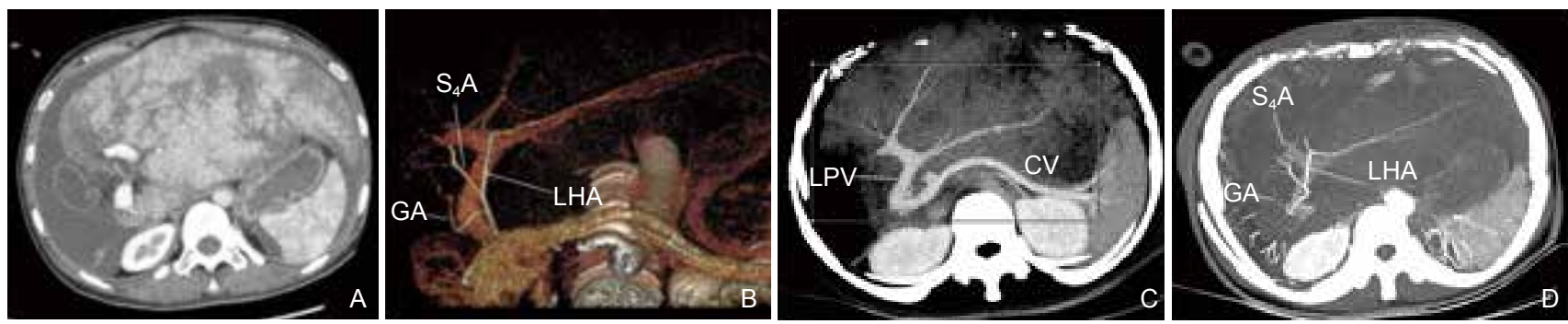

Figure 2 (A) Contrast-enhanced computed tomography showed no liver parenchyma on the right side of the gallbladder fossa, compensatory hypertrophy of left hepatic lobe and geographically distributed hepatic congestion during the portal venous phase; (B and D) Vascular reconstruction of CT imaging showed complete absence of the right hepatic artery, gallbladder artery originating from the left branch of hepatic artery and no replacement of the right hepatic artery from the superior mesenteric artery; (C and D) no right portal vein branch from the main portal trunk was noted. GA, gallbladder artery; LHA, left branch of hepatic artery; LPV, left branch of portal vein; SV, spleen vein.
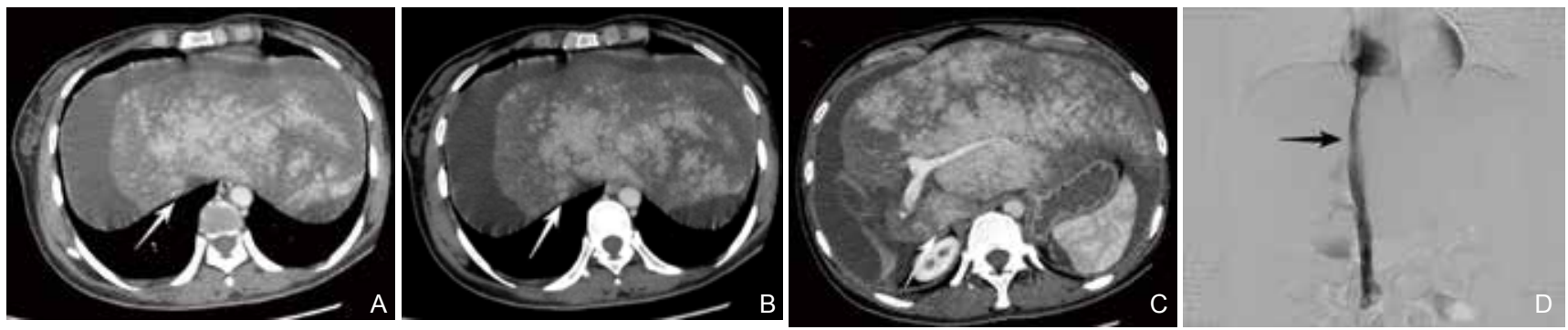

Figure 3 (A, B and C) The inferior vena cava of our case (white arrows) didn't pass through the liver parenchyma on any of the CT images but simply contacted with the posterior visceral surface of the left lobe. (D) There was no obvious stenosis of the inferior vena cava seen (black arrow). No obvious enhancement of the hepatic vein or its collateral branches were observed.

\section{Discussion}

Congenital agenesis of a main liver lobe is a rare malformation affecting the left hepatic lobe more often than the right lobe [1]. Anomalies of morphology related to developmental defects could be categorized as agenesis, aplasia and hypoplasia. Agenesis is the condition with absence of one lobe that is replaced by fibrous tissue. Aplasia is the condition that one of the lobes is small and its structure is abnormal, with few hepatic trabeculae, numerous bile ducts, and abnormal blood vessels. Hypoplasia is diagnosed when one of the lobes is small while its structure is normal [3]. The case we reported would be categorized as an agenesis of right hepatic lobe according to the above definitions. Inoue $\mathrm{T}$ et al. [8] reported a rare case of hypogenesis of the right lobe of the liver with portal hypertension and a review of 31 cases of agenesis or hypogenesis of the right hepatic lobe in Japan. They described the difference between agenesis and hypogenesis. Histopathologically, the agenesis contains the abundant connective tissue with a few hepatic trabeculae, numerous bile ducts, and many abnormal blood vessels. Whereas the hypogenesis has one small lobe, with normal hepatic structures [3]. Several studies $[2,3,5,8,9]$ described the ultrasonography and CT manifestations of agenesis of the right hepatic lobe, including compensatory hypertrophy of the left liver lobe or caudate lobe, colonic interposition between liver and diaphragm, gallbladder of ectopic position which was retrohepatic or suprahepatic below the right hemidiaphragm, absence of right hepatic artery, right portal vein branch or dilated right intrahepatic ducts. Chou et al. [2] established the diagnostic criteria for agenesis of hepatic lobes on CT scanning, that are the absence of visualization of the three intraparenchymal tubular structures: i.e., right hepatic vein, right portal vein with its branches, and dilated right intrahepatic ducts, in which our case was in accordance with this diagnosis criteria. Absence of the visualization of all these intraparenchymal structures in the liver is a prerequisite for the diagnosis of agenesis of the right hepatic lobe on CT. However, identification of any one of these structures excludes the possibility of 
diagnosis of agenesis. Differential diagnosis includes severe right hepatic atrophy secondary to liver cirrhosis, choledocholithiasis, cholangiocarcinoma, idiopathic portal hypertension, Caroli's disease, prior fulminant hepatitis and previous surgery resection.

Agenesis of the right hepatic lobe is considered to be caused by either a failure of the right portal vein development or a mutual induction error between the primitive diaphragm and the endodermal diverticulum which representing the primitive liver. Agenesis is always accompanied by diaphragmatic hernia caused by partial or complete absence of the right semi-diaphragm [5], intestinal malrotation [10] and/or choledochal cyst [5]. Tanematsu et al. [11] thought that the severe atrophy of the right hepatic lobe which could not be identified on radiologic imaging simply secondary to liver cirrhosis was very uncommon. Actually, to our knowledge, there are no reports analyzing the morphologic changes of cirrhotic livers with time and verifying acquired complete atrophy of the right hepatic lobe and resulting in retrohepatic gallbladder.

Normally, intrahepatic segment of the IVC passes through the liver parenchyma, surrounded by the parenchyma of caudate lobe at the level of the junction of hepatic veins to the inferior vena cava [12]. However, the inferior vena cava simply contacts with the posterior visceral surface of the left lobe at the level of the junction of hepatic veins to the inferior vena cava, which also reveals congenital right hepatic lobe agenesis. Our case was consistent with the latter disorder. Considering the diagnosis could not be confirmed by CT sectional scanning, angiography or vascular imaging reconstruction maybe strongly beneficial in establishing the diagnosis. Absence of visualization of right hepatic artery branch, right hepatic vein and right portal vein branch is a prerequisite for the diagnosis of right hepatic lobe agenesis on CT [13]. As is suggested in this case, vascular reconstruction of CT imaging was useful to show absence of right hepatic artery and right portal vein and its branches, gallbladder artery originating from the branch of left hepatic artery, and absence of right hepatic vein. Even though the patient had abdominal injury history, no surgery was performed and thus no pathological results was able to be obtained in this case. All imaging evidences supported the diagnosis of right hepatic lobe agenesis, instead of severe right hepatic lobe atrophy due to trauma.

To our knowledge, there was no report about congenital right hepatic lobe agenesis combined with Budd-Chiari Syndrome in literature so far. The entrance of the LHV to the inferior vena cava was detected on ultrasound but not on DSA. We assume the reason is that the LHV entrance of the was too narrow as shown on ultrasound, it was hard to put the guidewire through for imaging. The right hepatic lobe of this patient was absent, with compensatory hypertrophy of left hepatic lobe growing backward and rightward, and rotating clockwise along the hepatic pedicle. The $\mathrm{S}_{4} \mathrm{~V}$ was blocked without blood flow signal being detected at the secondary porta. Collateral vessels from the distal part of $\mathrm{S}_{4} \mathrm{~V}$ with reverse blood flow drained into the left hepatic vein could be detected. The left hepatic vein at the secondary porta was a continuous flat monophasic wave without normal periodic venous plasticity, with increased blood flow velocity which indicated stenosis. In our case, no blood flow of the $\mathrm{S}_{4} \mathrm{~V}$ was detected at the secondary porta during the ultrasonographic examination, but it could be detected with contrast-enhanced CT. We assumed the possible reason was that the blood flow was slow and could not be detected by conventional color Doppler imaging. We could not confirm whether there was a direct causal relationship between occlusion of the $\mathrm{S}_{4} \mathrm{~V}$ and stenosis of the left hepatic vein at the secondary porta and the clockwise rotation of liver along the hepatic pedicle.

\section{Conclusions}

Agenesis of the right hepatic lobe is conceptualized as a very rare anomaly. Patient's nonspecific symptom of right hepatic agenesis is of little clinical significance. The majority of patients with right hepatic agenesis usually remain concealed at early stage, but are often accompanied by clinical symptoms secondary to atypical cholecystitis, gastric volvulus or portal hypertension with age [13]. Thus, it should be pointed out that it is very important to understand this developmental anomaly of liver with complicated clinical presentation and outcome. Full awareness and profound knowledge of this developmental anomaly is of substantial necessity to avoid fatal complications during interventional and surgical treatment.

\section{Acknowledgements}

This work was supported by the Beijing Municipal Administration of Hospitals' Ascent Plan (DFL 20180102).

\section{Conflict of Interest}

The authors declare that they have no conflict of interest.

\section{References}

[1] Singh R. Hypoplastic left lobe of liver with accessory caudate lobe Case Rep Med 2013; 2013:604513.

[2] Chou CK, Mak CW, Lin MB, Tzeng WS, Chang JM. CT of agenesis and atrophy of the right hepatic lobe. Abdom Imaging 1998; 23: 603-7.

[3] Alicioglu B. Right liver lobe hypoplasia and related abnormalities. 
Pol J Radiol 2015; 13: 503-5.

[4] Matsushita K, Gotoh K, Eguchi H, Iwagami Y, Yamada D, Asaoka $\mathrm{T}$, et al. Agenesis of the left hepatic lobe undergoing laparoscopic hepatectomy for hepatocellular carcinoma: a case report. Surg Case Rep 2017; 3: 50.

[5] Radin DR, Colletti PM, Ralls PW, Boswell WD Jr, Halls JM. Agenesis of the right lobe of the liver. Radiology 1987; 164: 639-42.

[6] Van Wettere M, Bruno O, Rautou PE, Vilgrain V, Ronot M. Diagnosis of Budd-Chiari syndrome. Abdom Radiol (NY) 2018; 43: 1896-907.

[7] Nacif LS, Buscariolli Ydos S, D'Albuquerque LA, Andraus W. Agenesis of the right hepatic lobe. Case Rep Med 2012; 2012: 415742.

[8] Inoue T, Ito Y, Matsuzaki Y, Okauchi Y, Kondo H, Horiuchi N, et al. Hypogenesis of right hepatic lobe accompanied by portal hypertension: case report and review of 31 Japanese cases. $J$
Gastroenterol 1997; 32: 836-42.

[9] Kabaroudis A, Papaziogas B, Atmatzidis K, Argiriadou E, Paraskevas A, Galanis I, et al. Hypoplasia of the right hepatic lobe combined with a floating gallbladder. Acta Chir Belg 2003; 103: 425-7.

[10] Kovarik JL, Jensen NK. Congenital aplasia of the right hepatic lobe with right-sided diaphragmatic hernia and intestinal malrotation. Int Surg 1969; 51: 499-503.

[11] Kanematsu M, Imaeda T, Yamawaki Y, Doi H. Agenesis of the Right Lobe of the Liver: Case Report. Gastrointest Radiol 1991; 16: 320-2.

[12] Suneja SK, Teal JS. Scintigraphy in evaluation of the hypoplastic right hepatic lobe: a rare variant. J Natl Med Assoc 1989; 81: 209-11.

[13] Ceravolo I, Guerrieri D, De Vargas Macciucca M, De Cristofaro F, Panzironi G. MRI rare finding: Absence of the left liver lobe. Eur $J$ Radiol Open 2017; 14: 50-2. 